\title{
Assisting Dependent People at Home Through Autonomous Unmanned Aerial Vehicles
}

\author{
Lidia M. Belmonte ${ }^{1,2}$, Rafael Morales ${ }^{1,2}$, Arturo S. García ${ }^{1,2}$, Eva Segura ${ }^{1,2}$, \\ Paulo Novais ${ }^{3}$, and Antonio Fernández-Caballero ${ }^{1,2,4}$ \\ 1 Universidad de Castilla-La Mancha \\ Instituto de Investigación en Informática de Albacete, 02071-Albacete, Spain \\ ${ }^{2}$ Universidad de Castilla-La Mancha \\ Escuela Técnica Superior de Ingenieros Industriales, 02071-Albacete, Spain \\ 3 Universidade do Minho, Escola de Engenharia \\ Campus de Gualtar, 4710-057 Braga, Portugal \\ 4 CIBERSAM (Biomedical Research Networking Centre in Mental Health), Spain
}

\begin{abstract}
This work describes a proposal of autonomous unmanned aerial vehicles (AUAVs) for home assistance of dependent people. AUAVs will monitor and recognize human activities during flight to improve their quality of life. However, before bringing such AUAV assistance to real homes, several challenges must be faced to make them viable and practical. Some challenges are technical and some others are related to human factors. In particular, several technical aspects are described for AUAV assistance: (1) flight control, based on our active disturbance rejection control algorithm, (2) flight planning (navigation in obstacle environments), and, (3) processing signals, acquired both from flight-control and monitoring sensors. From the assisted person's viewpoint, our research focuses on three cues: (1) the user's perception about AUAV assistance, (2) the influence on human acceptance of AUAV appearance and behavior at home, and (3) the human-robot interaction between assistant AUAV and assisted person. Finally, virtual reality environments are proposed to carry out preliminary tests and user acceptance evaluations.
\end{abstract}

Keywords: autonomous unmanned aerial vehicles, home assistance, dependent people

\section{Introduction}

The use of unmanned aerial vehicles (UAVs) has notably increased in the last years. Moreover, computer vision in UAVs plays a role beyond serving as mere recording and displaying of flight environments. By means of computer vision algorithms, it is possible to extract useful information both of the aircraft's state and of its environment. This article proposes a framework for assisting dependent people at home through vision-based autonomous unmanned aerial vehicles (AUAVs) which do not require the presence of an operator and navigate indoor without contravening current laws for flying outdoors. Thus, the overall objective is to enhance dependent people's quality of life (QoL). QoL is the 
appreciation of well-being in daily human lives, including emotional, social and physical aspects. QoL of dependent people is usually reduced as a consequence of their functional incapacity for carrying normal daily activities. In addition, dependents often prefer to live in their own homes against other options, but it is difficult to provide the necessary security and home care without monitoring $[1$, 2 ]. In this sense, the combination of information and communication technologies with mobile robotics provides intelligent and proactive actions to most problems that dependent people suffer at home. Thus, they facilitate the care of dependent people so that they improve their QoL in the comfort of their proper homes.

It is our conviction that aerial vehicles can complement other technologies in assisting dependents at home. Indeed, AUAVs act alone or in combination with other technologies like surveillance cameras and biometric bracelets. They also reach out where other solutions do not (for example, blind zones) through accompanying the person, positioning itself to observe his/her activities, evaluating his/her emotional state, and acting in accordance to each situation. For this sake, the main challenges for AUAVs to assist dependents within a family environment are described throughout this article. Explicitly, a double engineering solution to put in practice the above mentioned objective is described.

In first place, we have detected important technical challenges in terms of flight control, flight planning in environments with obstacles and signal processing. The solution for robust flight at home is active control by disturbance rejection, our proper algorithm recently introduced [3]. Signal processing is required for sensors that control the flight itself (e.g. inertial measurement unit sensors) and also those that observe the habitat. Obviously, the observed environment includes the dependent so that an on-board camera will capture the person's activities and facial expressions.

Secondly, human factors must consider the individual not just as an obstacle, but respecting his/her personal space when calculating the flight paths. In addition, the potential prejudices and doubts that a person has towards a flying robot, probably considered as an intruder or threat, have to be saved. Lastly, the most appropriate human-robot interaction between assisted and assistant must be defined to build a relationship of trust.

Finally, such proposal has to be evaluated. In this initial stage, the focus is put towards the acceptance of AUAVs as assistants, deepening in the user's response in immersive virtual reality environments to AUAV appearance and behavior, as well as the interaction between human and robot.

\section{Technical Challenges}

The development of AUAVs to serve as assistants for dependents at their own homes requires the design of a solution for autonomous and safe flight of the aircraft. The flight environment, that is the dependent person's home, is initially a place containing static obstacles from the viewpoint of navigation. However, the dependent person also moves around the environment as a dynamic obstacle that must be followed by the AUAV. In this context, the use of multi-rotor 
AUAVs has been considered appropriate due to their excellent maneuverability, agility, and versatility.

In addition, it is mandatory to reduce the AUAV's size in order not to interfere with people's daily routines in limited home space. However, such reduction makes difficult its control. Mainly, the sensitivity of an AUAV is highly affected both by exogenous (wind gusts) and endogenous (large non-linearities, uncertainties, dynamic couplings, etc.) disturbances that seriously affect its flight capacity and stability [4]. Several works have been developed to reduce the effects of wind gusts on the AUAV [13], but most of them are based on the assumption of persistent gusts of wind with a fixed speed, a fact that almost never occurs in reality. There are also enormous limitations on the available space, payload, and capacity of an AUAV's power supply system, leading to the use of small processors with low power consumption and limited memory [5].

On the other hand, the integration of AUAVs within the dependent person's home must be based on the principle that safety is not compromised during flight, exhibiting a level of safety equivalent to that of manned flight missions [7,8]. All this makes it necessary to design robust control algorithms that can be implemented in real small-size AUAVs [9]. Unfortunately, conventional control methods (proportional integral derivative (PID) and linear quadratic (LQR)) present serious performance problems when the size of AUAVs is reduced [10]. Therefore several methods have been developed to improve the control performance in multi-rotor AUAVs. These are non-linear type controllers based on mathematical models obtained through using complicated non-linear models and identification methods [11]. Many of them present highly complex problems, which complicates their use in real AUAVs with low consumption processors and limited memory. Another disadvantage is that the multi-rotor AUAV's dynamic model is an approximation to the real system, showing therefore problems due to parametric uncertainties and noise in sensors' measures.

In addition, robust algorithms of an adaptive nature have been developed in recent years to address the problem of parametric uncertainties in AUAVs. Unfortunately, they present problems when the multi-rotor AUAV navigates in outdoor environments (including indoors with open windows), as these control systems are affected by external wind disturbances [12]. In this sense, the development of controllers for multi-rotor AUAVs performing aggressive maneuvers under disturbances due to variable speed wind is now a completely open field.

In addition, the measurements from sensors have a high noise component, especially when the sensors are low cost [6]. In fact, signals can cause the control system to become unstable when used in feedback or compensation loops. The search for methods to eliminate noise from signals through hardware and/or software is a field of current research in many application domains. In this way, a prevailing research field is to obtain signal filters that hardly present delays, have high robustness with respect to noise, do not need to presuppose statistical properties of noise, and can be implemented in low-cost hardware systems online and in real-time. Thus, a first major challenge for the development of our proposal is the design of innovative robust flight control and signal processing 


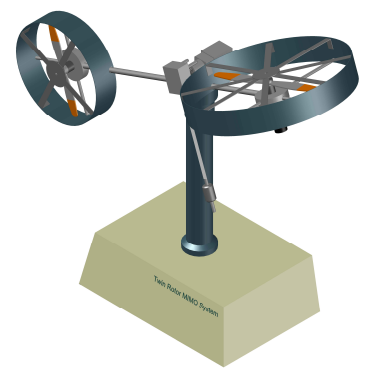

Fig. 1. TRMS system used in flight control simulations

systems to be implemented on small processors with reduced energy consumption and limited memory.

We propose to improve the efficiency and robustness of the AUAV against significant uncertainties in its modeling and external disturbances to solve this first challenge. This has been done through the development of new algorithms based on the concept of active disturbance rejection control (ADRC) [3] and its experimentation with the Twin Rotor MIMO System (TRMS) (see Fig. 1).

ADRC handles the effects of disturbances (endogenous and exogenous) as an aggregate, global, purely time-dependent function without a particular structure. This concept allows the designer to avoid the use of an observer based on the non-linear structure of the (often non-existent) system and proposes a non-linear injection module of the inputs through their gain factors, instead of a merely linear observer. The arbitrarily close estimate of the aggregate disturbance allows its approximate cancellation through the appropriate control action. With this new concept it is intended to attain: (a) an improvement in the efficiency of the AUAV behavior, (b) a higher tolerance to large disturbances in the AUAV, (c) an easier adjustment and operation, and, (d) a drastic reduction of the AUAV probability to go into loss when performing home monitoring tasks.

\section{$3 \quad$ Human Factor Challenges}

Automated monitoring and identification of humans is a valuable tool in many areas such as rehabilitation, clinical psychology and gerontology for taking care in the family environment [14]. The availability of new static and mobile sensor types, and the consequent fusion of multi-sensory data in monitoring tasks offers novel solutions to model environments and diagnose situations based on the analysis of sensory data sequences [15].

Monitoring people physical activities and behaviors from computer vision is now well-established in our team [16]. For recognizing the emotional state of the human, a non-intrusive process is automatic detection of emotions based on the study of facial expressions [17]. The Facial Action Coding System encodes all possible facial expressions according to action units (AUs) that occur individually or in combination [18]. The exploitation of these context-aware emotional 
devices allows to deliver a highly personalized and dedicated collection of services designed to support users and improve their personal care.

Thus, the AUAV performs a capture of images of the dependent person's face. This requires the detection and focus on the principal parts of the face (eyes, nose, mouth, etc.). All this requires the positioning of the AUAV in front of the person's face from time to time. The captured information is sent to a base station responsible for the recognition of the dependent's emotions. In this way, the system evaluates the person's emotional state, together with his/her behavior, to determine the assistance needed for each situation.

Let us highlight that the ability of an UAV to fly autonomously is essential to carry out the proposed monitoring tasks. This ability is mandatory when considering that homes are closed environments composed of static and dynamic obstacles of different sizes and types, which endangers the safety of the monitored people. In the scientific literature, there are two approaches to autonomous navigation. (a) In deliberative motion planning the trajectories are obtained assuming a global knowledge of the environment, that is, a static environment [19]. In general, a deliberate trajectory planner is useful when the environment is known a priori, but may require too much computational effort when the environment is dynamic. (b) Reactive motion planning considers obstacle detection by means of a local sensory system (laser, optical flow sensors, stereo cameras or a single camera) and the consequent control of the trajectory to avoid the detected obstacles [20]. In this case, the information is incomplete and uncertain, and suffers from the additional problem of the difficult specification of direct movement plans.

In this sense, we propose to improve the navigation of the AUAV in environments with static and dynamic obstacles by developing a trajectory planner that takes into account the AUAV's dynamic and energetic constraints, and that contemplates the appearance of unexpected obstacles through computer vision and its corresponding integration in the AUAV control algorithm. In this respect, a navigation system composed of different stages is proposed. In the first stage it is assumed that the physical navigation environment is known (map of the home). Several methodologies for obtaining trajectories will be studied by means of optimization algorithms that result in AUAV trajectories free of obstacles. During the second stage new algorithms will be developed to provide the AUAV with the ability to adapt to dynamic environments by integrating the information coming from the sensory system to monitor the dependent person at home.

An interesting example on trajectory planning has been presented for autonomously monitoring wind turbine blades by means of a quadrotor following a hyperbolic path around the blade [21]. UAVs for monitoring large and inaccessible structures have been also used by health, safety and environment inspectors in complex constructions such as power stations [22]. Recently, a novel framework has been developed to increment the ability of autonomous navigation, especially in cluttered environments [23]. Hence, robust and fast motion close to obstacles has been achieved, which demonstrates that a deeper integration 


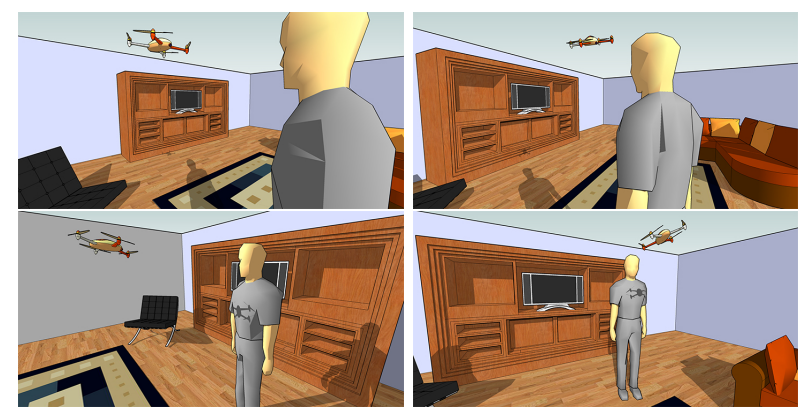

Fig. 2. VR environment to simulate AUAV assistance

of motion planning and perception improves robustness and computational efficiency. Future flight safety improvements will allow to extent the use of AUAVs in complex environments such as cities.

\section{Virtual Reality Validation Process}

The research work in AUAV assistance requires the validation of the technology and the acceptance of the dependent people. The obtained conclusions will allow to improve further developments. The validation should be carried out at homes of people willing to participate. Nevertheless, in this initial stage of development, it has been considered more appropriate to use simulations. This option saves costs, is more versatile and, most important, is much safer.

For this, we rely on virtual reality (VR) to perform evaluations, focused both on the flying robot assistant and on the assisted person. Thus, virtual worlds will be generated to process the sensor signals and evaluate the progress in flight control and planning. Virtual environments will recreate the dependent and his/her home (a closed environment with obstacles). The behavior of the AUAV will be simulated in different scenarios (see Fig. 2) using MATLAB and Unity $3 \mathrm{D}$.

Regarding a user validation of the proposal, the focus should be towards the acceptance of AUAVs as assistants, deepening in the users' responses to AUAV appearance and behavior, and human-robot interaction. Through the inclusion of immersive VR headsets and semi-immersive VR technologies, it is possible for real people to experience first hand sharing their space with AUAVs assistants. Even more, VR allow to observe the reaction of people to the flight of different virtual AUAVs around them and to investigate on several human-robot interaction techniques.

\section{Conclusions}

Personal dependency is defined as a functional incapacity for the development of daily-life activities, which requires assistance for their realization. However, 
dependent people usually prefer to live at their own homes, which implies care strategies in the family environment. This paper has proposed a solution for the development of indoor AUAV-based systems that allow home assistance of dependents with the aim of improving their quality of life.

Concretely, the use of AUAVs based on computer vision is proposed for the support and help of dependents. This proposal aims to develop UAVs capable of flying autonomously in a home to perform the task of monitoring and assisting dependents. However, this development process entails multiple challenges, both technical and human, that have to be addressed before making possible the use of AUAVs as assistants at home (e.g. a safety radius must be considered during the whole monitoring process to avoid collisions between UAV and person). In this article, we have introduced the bases for the development of solutions to advance in the line of research proposed. We have considered the aspects of AUAV control and navigation, as well as human monitoring. Lastly, virtual reality has been proposed as a key element in the validation of the robotic assistance system.

\section{Acknowledgments}

This work has been partially supported by Spanish Ministerio de Ciencia, Innovación y Universidades, Agencia Estatal de Investigación (AEI) / European Regional Development Fund (FEDER, UE) under DPI2016-80894-R grant, and by CIBERSAM of the Instituto de Salud Carlos III. Lidia M. Belmonte holds FPU014/05283 scholarship from Spanish Ministerio de Educación y Formación Profesional.

\section{References}

1. A. Fernández-Caballero, A. Martínez-Rodrigo, J.M. Pastor, J. C. Castillo, E. Lozano-Monasor, M. T. López, R. Zangróniz, J. M. Latorre, and A. FernándezSotos, "Smart environment architecture for emotion recognition and regulation," J. Biomed. Inform., 64, 55-73, 2016.

2. J. C. Castillo, Á. Castro-González, A. Fernández-Caballero, J. M. Latorre, J. M. Pastor, A. Fernández-Sotos, and M. A. Salichs, "Software architecture for smart emotion recognition and regulation of the ageing adult," Cogn. Comput., 8(2), 357-367, 2016.

3. L. M. Belmonte, R. Morales, A. Fernández-Caballero, and J. A. Somolinos, "A tandem active disturbance rejection control for a laboratory helicopter with variable speed rotors," IEEE Trans. Ind. Electron., 63(10), 6395-6406, 2016.

4. R. Mahony, V. Kumar, and P. Corke, "Multirotor aerial vehicles: Modeling, estimation, and control of quadrotor," IEEE Robot. Autom. Mag., 19(3), 20-32, 2012.

5. R. C. Leishman, J. C. MacDonald, R. W. Beard, and T. W. McLain, "Quadrotors and accelerometers: State estimation with an improved dynamic model," IEEE Control Syst. Mag., 34(1), 28-41, 2014.

6. M. H. Tanveer, S. F. Ahmed, D. Hazry, F. A. Warsy, and M. K. Joyo, "Stabilized controller design for attitude and altitude controlling of quad-rotor under disturbance and noisy conditions," Am. J. Appl. Sci., 10(8), 819-831, 2013. 
7. L. M. Belmonte, R. Morales, A. Fernández-Caballero, and J. A. Somolinos, "Robust decentralized nonlinear control for a twin rotor MIMO system," Sensors, 16(8), 1160, 2016.

8. Y. Yu, G. Lu, C. Sun, and H. Liu. "Robust backstepping decentralized tracking control for a 3-DOF helicopter," Nonlinear Dynam., 82(1-2), 947-960, 2015.

9. A. Fernández-Caballero, L. M. Belmonte, R. Morales, J. A. Somolinos, "Generalized proportional integral control for an unmanned quadrotor system," Int. J. Adv. Robot. Syst., 12, 85, 2015.

10. S. Bouabdallah, A. Noth, and R. Siegwart. "PID vs LQ control techniques applied to an indoor micro quadrotor," in Proc. 2004 IEEE/RSJ Int. Conf. Intell. Robot. Syst., Senday, Japan, 2004), 2451-2456.

11. S. Bertrand, N. Guenard, T. Hamel, H. Piet-Lahanier, and L. Eck, "A hierarchical controller for miniature VTOL UAVs: Design and stability analysis using singular perturbation theory," Cont. Eng. Pract., 19(10), 1099-1108, 2011.

12. Z. T. Dydek, A. M. Annaswamy, and E. Lavretsky, "Adaptive control of quadrotor UAVs: A design trade study with flight evaluations," IEEE Trans. Cont. Syst. Tech., 21(4), 1400-1406, 2013.

13. L. Sun, and Z. Zuo, "Nonlinear adaptive trajectory tracking control for a quadrotor with parametric uncertainty," Proc. Inst. Mech. Eng. G, 229(9), 1-13, 2014.

14. J. C. Castillo, Á. Castro-González, F. Alonso-Martín, A. Fernández-Caballero, and M. A. Salichs, "Emotion detection and regulation from personal assistant robot in smart environment," in Personal Assistants: Emerging Computational Technologies, A. Costa, V. Julián, and P. Novais, Eds. New York: Springer, 2018), 179-195.

15. R. Morales, A. Fernández-Caballero, J. A. Somolinos, and H. Sira-Ramírez, "Integration of sensors in control and automation systems," J. Sensors, 2017, 6415876, 2017.

16. J. C. Castillo, A. Fernández-Caballero, J. Serrano-Cuerda, M. T. López, A. Martínez-Rodrigo, "Smart environment architecture for robust people detection by infrared and visible video fusion," J. Ambient. Intell. Humaniz. Comput., 8(2), 223-237, 2017.

17. E. Lozano-Monasor, M. T López, F. Vigo-Bustos, and A. Fernández-Caballero, "Facial expression recognition in ageing adults: From lab to ambient assisted living," J. Ambient. Intell. Humaniz. Comput., 8(4), 567-578, 2017.

18. P. Ekman, W. V. Friesen, and J. C. Hager, Facial Action Coding System. Manual and Investigator's Guide. Salt Lake City, UT: Research Nexus, 2002.

19. F. Lamiraux, S. Sekhavat, and J. P. Laumond, "Motion planning and control for Hilare pulling a trailer," IEEE Trans. Robot. Autom., 15(4), 640-652, 1999.

20. T. Balch, and R. C. Arkin, "Behavior-based information control for multirobot teams," IEEE Trans. Robot. Autom, 14(6), 926-939, 1998.

21. S. Shivaram, "Structural Health Monitoring of Wind Turbine Blades using Unmanned Air Vehicles," (Master's Dissertation, University of Dublin) 2015.

22. H. Whitemore, "Koweit : how a drone is being used to monitor Health \& Safety at the construction site," ENGIE Innovation. Retrieved from: https://innovation.engie.com/en/news/news/smart-buildings/koweit-how-adrone-is-being-used-to-monitor-health-safety-at-the-construction-site-1/1112 2015.

23. P. R. Florence, J. Carter, J. Ware, R. and Tedrake, "NanoMap: Fast, UncertaintyAware Proximity Queries with Lazy Search over Local 3D Data," In International Conference on Robotics and Automation (ICRA), Brisbane, Australia. 2018. 